\title{
Uma Revisão Sistemática sobre Abordagens em Organizações Virtuais no Contexto de Confiança, Reputação e Computação em Nuvem
}

\section{A Systematic Review on Approaches in Virtual Organizations in the Context of Trust, Reputation, and Cloud Computing}

\author{
Luís Felipe Bilecki ${ }^{1}$ \\ Marcelo da Silva Hounsell ${ }^{2}$, Adriano Fiorese ${ }^{2}$
}

Data de submissão: 08/11/2016, Data de aceite: 01/05/2017

\begin{abstract}
Resumo: As Organizações Virtuais representam uma proeminente forma de colaboração na qual um conjunto de empresas compartilham habilidades, competências e riscos para atender um objetivo em comum. Algumas preocupações surgem durante a colaboração, como, por exemplo, questões relativas a confiança, reputação e o uso de recursos tecnológicos. A confiança e a reputação nas OVs visam minimizar o risco ao lidar com entidades desconhecidas. Já o uso de recursos tecnológicos, como por exemplo computação em nuvem, fornece suporte a oportunidade de colaboração porém apresenta alguns problemas relacionados a autenticação, segurança, privacidade e confiança. Portanto, este artigo apresenta uma revisão sistemática cujo objetivo é verificar as abordagens que estão sendo realizadas no contexto das Organizações Virtuais e que estejam relacionadas com pelo menos um dos conceitos de confiança, reputação ou computação em nuvem. Para tal, a busca junto a literatura especializada resultou em 75 artigos, sendo selecionadas 24 deles para análise. Os critérios utilizados para a seleção foram os artigos relevantes nos últimos 5 anos, completos, no idioma inglês e que relacionassem Organização Virtual com pelo menos um dos conceitos de confiança, reputação e computação em nuvem. Por conseguinte, identificou-se como cada conceito é abordado e verificou-se que um mesmo conceito apresenta-se de diferentes formas e nenhum dos trabalhos buscou apresentar a integração desses conceitos.
\end{abstract}

\footnotetext{
${ }^{1}$ Programa de Pós-Graduação em Computação Aplicada, Centro de Ciências Tecnológicas, Universidade do Estado de Santa Catarina, Caixa Postal 631 - Joinville, Santa Catarina, Brasil

\{luis.bilecki@gmail.com\}

${ }^{2}$ Departamento de Ciência da Computação (DCC), Programa de Pós-Graduação em Computação Aplicada, Centro de Ciências Tecnológicas, Universidade do Estado de Santa Catarina, Caixa Postal 631 - Joinville, Santa Catarina, Brasil

\{marcelo.hounsell@udesc.br, adriano.fiorese@udesc.br\}
} 
Uma Revisão Sistemática sobre Abordagens em Organizações Virtuais no Contexto de Confiança, Reputação e Computação em Nuvem

\begin{abstract}
Virtual organizations represent a prominent form of collaboration, in which a number of companies share skills, competencies and risks to address a common goal. Some issues arise during the collaboration, such as trust, reputation, and the use of technological resources. The trust and reputation in VO's aim to reduce the risks during the transaction with unknown parties. Also, the use of technological resources (e.g. cloud computing) brings support to the collaboration opportunity, however presents issues related to authentication, security, privacy and trust. Thus, this article presents a systematic review, whose objective is to verify the approaches that are being in virtual organization context and are related to at least one of the concepts of trust, reputation or cloud computing. For this purpose, the search along the specialized literature has resulted in 75 articles, where 24 of them were selected for analysis. The selection criteria are used to filter search result, such as papers with 4 pages or more, articles published in the last five years, written in English Language and correlating virtual organization with at least one of that concepts. It was identified that each concept is treated and it was found that the same concept is presented in different ways and none of the studies accomplished to present the integration of these concepts.
\end{abstract}

Palavras-chave: Organizações virtuais, confiança, revisão sistemática.

Keywords: Virtual organizations, trust, systematic review.

\title{
1 Introdução
}

Os diversos desafios sócio-econômicos impostos pela sociedade, como a globalização, competitividade, baixo custo na produção e a variedade de produtos e serviços, motivaram o surgimento de diversas redes de colaboração [1]. As empresas têm se especializado e colaborado umas com outras para sobreviver à competitividade imposta pelas empresas concorrentes no mercado. Através da colaboração, as empresas se unem para atingir um objetivo em comum, com o propósito de reduzir custos, aumentar a qualidade dos produtos, entre outros. À vista disto, o processo de colaboração tem sido facilitado devido aos avanços proporcionados na área de tecnologia de informação, fornecendo meios para interconectar entidades geograficamente dispersas [2].

As Organizações Virtuais (OVs) representam uma proeminente forma de colaboração, sendo vantajosa ao incorporar flexibilidade e dinamismo para tratar as demandas de mercado. A OV configura-se como uma aliança temporária na qual um conjunto de entidades (geralmente empresas) legalmente independentes, heterogêneas e geograficamente dispersas compartilham recursos, habilidades e riscos para atender a uma oportunidade de colaboração 
Uma Revisão Sistemática sobre Abordagens em Organizações Virtuais no Contexto de Confiança, Reputação e Computação em Nuvem

[2]. Além disto, as OVs podem usar as tecnologias de informação e comunicação, por exemplo, Computação em Nuvem (CN) e Grid Computing, para suportar os negócios e transações entre as pessoas e as empresas.

Neste contexto, os membros (empresas) da OV devem compartilhar, combinar e integrar informações em um ambiente em que as maiores preocupações são a confiança e a segurança [3]. Um dos obstáculos para o atendimento da oportunidade de colaboração é o estabelecimento e gerenciamento da confiança entre os envolvidos, o qual no ambiente de organizações virtuais, configura-se como um fator crítico [4]. A confiança pode ser entendida como uma probabilidade subjetiva pela qual um indivíduo, A, espera que outro elemento, B, exerça uma ação de acordo com o seu comportamento [5]. Já no ambiente da OV, ela pode ser entendida como um valor numérico que auxilia o gestor da OV a tomar a decisão de cooperar ou colaborar com um determinado parceiro de negócio ou provedor de serviço que está sendo avaliado [6].

Outro conceito estritamente relacionado com a confiança é a reputação. A reputação é definida como uma coleção de avaliações passadas a respeito dos participantes de uma comunidade, auxiliando as pessoas na escolha de parceiros confiáveis no mundo virtual que são honestos no mundo real [7]. Sendo que, uma das alternativas para se alcançar o estabelecimento e gerenciamento da confiança em ambientes como o das organizações virtuais, é através de sistemas de reputação. A utilização de tais sistemas apresenta benefícios ao lidar com entidades desconhecidas, pois auxilia na garantia de confiança na relação entre os candidatos que vão formalizar uma OV, em que a reputação é vista como uma combinação de indicadores de desempenho, colaboração e recomendações passadas.

Além dos problemas de confiança e reputação existentes no cenário das OVs, o uso de recursos tecnológicos (computação em nuvem, por exemplo) também desperta preocupações, pois os parceiros de negócio da OV disponibilizam seus serviços por meio dos recursos fornecidos pelos provedores de nuvem computacional. Desta forma, devem confiar no serviço e consequentemente na qualidade do serviço fornecida. Além destes problemas, como a OV é um ambiente dinâmico, outras necessidades relativas a autenticação e segurança motivam abordagens de pesquisa.

Com todos estes conceitos e problemas abordados em organizações virtuais, torna-se relevante realizar uma revisão sistemática para identificar quais abordagens estão sendo realizadas em organizações virtuais e que estejam relacionadas com confiança, reputação ou o uso de recursos tecnológicos para facilitar o processo de colaboração. Ainda, tal revisão é oportuna no sentido de também verificar a ocorrência de contribuição que apresente a combinação desses três conceitos bem como analisar de que forma cada conceito é tratado e em que sentido pode auxiliar o ambiente das OVs.

Este artigo visa apresentar uma revisão sistemática objetivando identificar e analisar 
Uma Revisão Sistemática sobre Abordagens em Organizações Virtuais no Contexto de Confiança, Reputação e Computação em Nuvem

trabalhos que tratem da utilização de sistemas de confiança ou reputação em ambientes de organizações virtuais e se relacionem com computação em nuvem.

O restante deste artigo está estruturado da seguinte forma: A Seção 2 detalha o conceito de redes de colaboração e organizações virtuais. A Seção 3 detalha os conceitos de confiança, reputação e quais são as suas aplicações em uma rede de colaboração, como as organizações virtuais. Na Seção 4 são apresentadas a definição de computação em nuvem e o modelo padronizado pelo National Institute of Standards and Technology (NIST) [8]. Ainda, é apresentado qual é o relacionamento existente entre computação em nuvem e organizações virtuais. A Seção 5 apresenta as revisões relacionadas. Na Seção 6 é exposto o estudo de revisão adequado ao contexto do trabalho bem como os métodos que possibilitam a realização do processo de revisão. A Seção 7 expõe a síntese dos resultados obtidos através da revisão sistemática. Por fim, na Seção 8 é apresentada a conclusão dessa pesquisa.

\section{Organizações Virtuais}

Em virtude da competitividade do mercado atual, as empresas tradicionais se unem em redes colaborativas, compartilhando competências distintas para alcançar um objetivo em comum. Conforme Camarinha-Matos et al. [2], as redes colaborativas configuram-se a partir de uma grande diversidade de formas. Dentre elas, pode-se citar: uma clássica cadeia de suprimentos, caracterizada pela estabilidade e excelente definição de papéis, proporcionando o mínimo possível em coordenação e troca de informações ou uma Organização Virtual (OV), que é configurada como uma rede dinâmica e orientada a objetivos criada para atender um único projeto ou oportunidade de colaboração [9].

Uma OV é um tipo de rede de colaboração, em que um conjunto de entidades (geralmente empresas) legalmente independentes e heterogêneas se unem para compartilhar competências, recursos, riscos, custos e habilidades, a fim de alcançar objetivos específicos de negócio (atender uma oportunidade de colaboração) [2]. Uma característica marcante e determinante para OVs é o uso de recursos fornecidos pelas tecnologias da informação e comunicação (TIC) para obter vantagem competitiva, configurando-se como uma entidade única por meio da união das competências essenciais de seus membros, que podem ser instituições, empresas ou pessoas especializadas [10].

Devido a dinamicidade da aliança formada pelos membros da OV, torna-se interessante compreender o ciclo de vida de uma organização virtual e suas etapas. O ciclo de vida é composto por quatro estágios: criação, operação, evolução e dissolução [2]. Este modelo de organização é fortemente atrelado ao uso de TICs para dar suporte as suas operações e responder a oportunidade de colaboração de uma forma mais competitiva. Além da fase final de dissolução em uma rede colaborativa de organizações, aborda-se também uma possível etapa de metamorfose, na qual ocorrem mudanças na organização, dissolvendo-se a rede anterior e 
Uma Revisão Sistemática sobre Abordagens em Organizações Virtuais no Contexto de Confiança, Reputação e Computação em Nuvem

criando-se uma totalmente nova [2]. O ciclo de vida de uma OV é ilustrado pela Figura 1

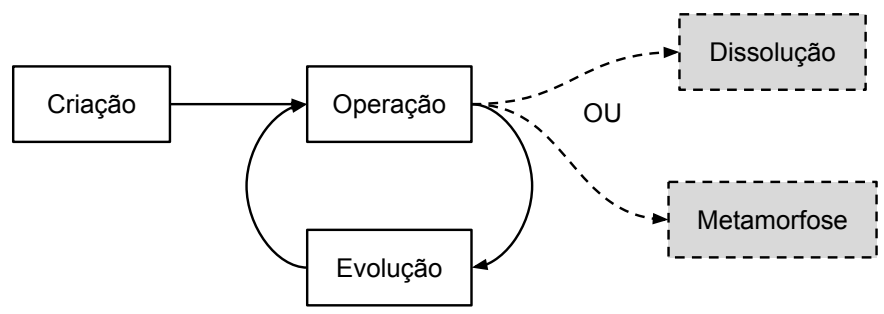

Figura 1. Ciclo de Vida das OVs. Adaptado de [2]

Conforme Camarinha-Matos [2], o modelo de ciclo de vida da OV é subdivido nas seguintes etapas:

- Criação: Processo inicial em que a OV é criada, configurada e todos os seus objetivos, pessoas ou entidades envolvidas são explicitados. Neste processo ocorrem as subfases: Configuração dos Recursos de Infraestrutura; Seleção de Parceiros de Negócio, Formação da OV e a Negociação e Contrato.

- Operação: Processo em que as entidades estão colaborando para atender a um objetivo em comum, ou seja, o fornecimento de um produto ou serviço. Dentro desta etapa, algumas atividades são necessárias, como troca de informações, coordenação avançada e funções relacionadas aos produtos/serviços.

- Evolução: Ocorre quando a OV já está em operação e torna-se necessário adicionar ou substituir um parceiro de negócio devido à incapacidade ou a necessidade de aumento de tarefas. Para esta etapa são necessárias as mesmas atividades descritas na etapa de Criação, para que a OV possa ser reconfigurada;

- Dissolução: É ocasionada pelo término das atividades de uma OV, ou seja, a entrega do produto/serviço ao cliente. Desta forma, pode ser motivada por duas situações: a realização bem-sucedida de todos os seus objetivos ou pela decisão dos parceiros de negócio em interromper a operação. Nesta fase ocorrem as atividades de avaliação do desempenho dos parceiros, coleta das informações das avaliações, redefinição das responsabilidades de cada membro da OV e a redefinição ou interrupção dos direitos de acesso à informação depois de atingir a etapa de Evolução.

- Metamorfose: Mudança nos objetivos iniciais (ex.: princípios e filiação) afetando a rede de colaboração como um todo, sendo necessário novamente a execução de todos os passos descritos na fase de Criação da OV. Geralmente é motivada por uma mudança no 
Uma Revisão Sistemática sobre Abordagens em Organizações Virtuais no Contexto de Confiança, Reputação e Computação em Nuvem

mercado, em que a OV precisa adaptar ou alterar seu perfil competitivo ou é ocasionada pela adaptação à demanda do cliente, em que a OV se responsabiliza por atender novas competências.

Um dos obstáculos para a colaboração/cooperação é o estabelecimento e gerenciamento da confiança entre as partes envolvidas (empresas), configurando-se como um fator crítico para o sucesso no atendimento da oportunidade de colaboração [11,3]. A confiança está relacionada com o ciclo de vida da OV, em que na fase de Criação as empresas podem ser selecionadas com base na confiança reportada por outros membros; na fase de Operação ocorre o gerenciamento e manutenção da confiança e, na Dissolução, onde cada membro deixa seu comentário/avaliação sobre a transação realizada [12]. Uma das sugestões para estreitar a confiança no ambiente colaborativo, é a aplicação dos conceitos de reputação aliado a confiança, por meio de um sistema de reputação, que alavanca a confiabilidade entre os membros da rede colaborativa [13]. Sendo assim, na Seção 3, os conceitos de confiança e reputação são detalhados e sua relação com a OV é explicitada.

\section{Confiança e Reputação}

A confiança é entendida como um fator crucial em um ambiente dinâmico como as organizações virtuais, pois os membros necessitam compartilhar, integrar e combinar dados e informações. Formar uma OV confiável representa desafios, devido a heterogeneidade dos participantes, a falta de uma forma padronizada de estabelecimento de confiança e as próprias limitações da OV, como por exemplo a localização [3].

O conceito de confiança é originário das ciências sociais, responsável pelo estudo do comportamento dinâmico da sociedade humana. É um conceito multidisciplinar, sendo estudado e aplicado na psicologia, sociologia, economia e tecnologia. Os Psicólogos estudam a confiança como uma atitude mental e focam nas ações ocorridas no cérebro da pessoa quando ela confia ou desconfia de alguém. Sociologistas abordam a confiança como um relacionamento social entre as pessoas, e os economistas definem a confiança como um termo de utilidade [14].

Além de ser um conceito multidisciplinar, configura-se como um conceito complexo que não apresenta uma definição universalmente aceita, sendo que cada área pode apresentar sua definição. Quando utiliza-se de recursos provenientes da nuvem computacional, pode-se definir a confiança como a percepção que o consumidor tem sobre o serviço disponibilizado pelo provedor de nuvem computacional [15]. Segundo o dicionário Merrian-Webster ${ }^{3}$, a confiança pode ser descrita como uma crença de que alguém ou algo é confiável, bom, honesto, eficaz, entre outros. Em uma implementação, por exemplo, comércio eletrônico, a confiança

${ }^{3}$ http://www.merriam-webster.com 
Uma Revisão Sistemática sobre Abordagens em Organizações Virtuais no Contexto de Confiança, Reputação e Computação em Nuvem

pode ser discutida como um valor numérico que indica o quão confiável um agente ou uma entidade é [16].

A reputação e a confiança são ferramentas de apoio a tomada de decisão, que reduzem o índice de riscos nas transações e interações em ambientes distribuídos. A reputação, um conceito próximo a confiança, pode ser definida como uma coleção de comentários (feedbacks) a respeito do comportamento passado dos participantes de uma comunidade [17]. Em ambientes como as OVs, torna-se difícil formalizar as relações pessoais. Muitas vezes, tornase necessário cooperar/colaborar com entidades (ex: empresas) com as quais ainda não houve experiências, desconhecendo-se assim o desempenho passado. Contudo, é possível caracterizar outros tipos de relação, particularmente estabelecidas entre as empresas. Os sistemas de reputação auxiliam na garantia de confiança no relacionamento entre esses candidatos para formarem uma $\mathrm{OV}$, em que a reputação pode ser entendida como uma combinação de indicadores de desempenho, colaboração e recomendações passadas (caso existam).

Dessa forma, com o objetivo de aumentar a sua abrangência diante do mercado competitivo, as organizações estão interessadas em trazer para perto outras entidades para cooperar e/ou colaborar. Um dos obstáculos observados no processo de colaboração é o estabelecimento e o gerenciamento da confiança entre as entidades envolvidas [11]. Compreendendo a confiança como um fator crítico que pode afetar a colaboração, Pan et al. [13] destacam que para resolver o problema ou buscar melhorar a interação e a confiabilidade entre os membros da OV, é desejável aplicar um sistema de reputação neste ambiente.

\section{Computação em Nuvem}

Com o rápido desenvolvimento da capacidade de processamento, armazenamento e a crescente utilização da Internet, os recursos computacionais tornaram-se mais baratos, poderosos e acessíveis de qualquer lugar. Esse incentivo da tecnologia proporcionou o surgimento de um conceito, a Computação em Nuvem, na qual os recursos computacionais na maioria das vezes são disponibilizados como um serviço.

A Computação em Nuvem não é um conceito novo, pois na década de 1960 John McCarthy já visualizava que a computação seria fornecida como serviço público (assim como a energia elétrica) e somente a partir da definição do modelo de negócios para o fornecimento de serviços sobre a Internet, pela Google, o termo popularizou-se [18]. O National Institute of Standards and Technology (NIST) [8] padronizou as definições e outros elementos presentes na computação em nuvem.

De acordo com o NIST [8], a computação em nuvem pode ser definida como um modelo para permitir o acesso via rede de forma ubíqua, conveniente e sob demanda a um agrupamento compartilhável e confiável de recursos computacionais (por exemplo redes, servido- 
Uma Revisão Sistemática sobre Abordagens em Organizações Virtuais no Contexto de Confiança, Reputação e Computação em Nuvem

res, armazenamento, aplicações e serviços). Estes recursos podem ser rapidamente liberados e fornecidos, sem qualquer intervenção humana, sendo que os recursos são provisionados de acordo com a necessidade do usuário [8]. O modelo padronizado pelo NIST apresenta cinco características essenciais, sendo elas: amplo acesso a rede, conjunto de recursos, acesso sob demanda, rápida elasticidade e serviço mensurável. Além disto, incorpora três modelos de serviço e quatro modelos de implantação.

$\mathrm{O}$ ambiente de nuvem computacional permite o fornecimento de serviços classificados em três modelos, referindo-se na forma como os serviços são entregues ao usuário final. Esses modelos de serviço incluem: (a) Software as a Service (SaaS): execução de aplicações na nuvem computacional (ex.: Google Docs); (b) Platform as a Service (PaaS): são fornecidas ferramentas de desenvolvimento de software, permitindo ao usuário/cliente criar suas aplicações utilizando a infraestrutura do provedor; (c) Infrastructure as a Service (IaaS): são fornecidos os recursos da infraestrutura computacional, como rede, armazenamento e processamento [8].

A nuvem computacional é baseada em quatro modelos de implantação com características e necessidades específicas para os usuários, podendo ter infraestrutura fornecida através dos seguintes modelos: (a) Pública: provedores de serviço fornecem sua infraestrutura como serviço para o público em geral; (b) Privada: toda a infraestrutura e recursos computacionais são de uso exclusivo da própria organização que a implantou e a mantém; (c) Híbrida: união de dois ou mais modelos, em que parte do serviço usa os recursos de uma nuvem privada e o restante usa os recursos providos por uma nuvem pública; (d) Comunitária: a infraestrutura é compartilhada entre as organizações que formam uma comunidade, na qual os recursos atendem as exigências dos membros do grupo (requisitos governamentais, educacionais, entre outros) [18].

As OVs podem utilizar estas tecnologias como forma de suporte ao atendimento da oportunidade de colaboração. Uma dessas tecnologias é a Computação em Nuvem (CN), com a qual a OV busca aumentar sua eficiência e flexibilidade, enquanto busca diminuir custos e o tempo de resposta. Pode-se perceber que alguns trabalhos apresentam a questão da integração da CN com OV [19, 20, 21, 22], já outros tratam dos problemas de autenticação e segurança existentes nestes ambientes [23] e, por fim, alguns trabalhos apresentam abordagens que fornecem suporte para a criação ou gerenciamento de OVs em plataformas de nuvem computacional (ex.: OpenStack) $[24,25,26]$.

\section{Revisões Relacionadas em OVs}

As pesquisas abordando as redes colaborativas envolvem muitas áreas, como engenharia, computação, psicologia e gerenciamento de negócios. Também abordam diversos conceitos sobre diferentes visões. Além disto, algumas revisões da literatura abordam temas 
Uma Revisão Sistemática sobre Abordagens em Organizações Virtuais no Contexto de Confiança, Reputação e Computação em Nuvem

específicos, como por exemplo identificação de características para OVs, revisão de metodologias, ferramentas e métodos aplicáveis a OV e revisão de uma aplicação de tecnologia em OV.

Nami [27] propõe uma revisão sobre o conceito de OV, apresentando definições, benefícios, propriedades e os desafios envolvidos na criação da OV, por exemplo, gerenciamento de confiança, segurança, autenticação e engenharia de ontologia.

Esposito e Evangelista [1] apresentam uma revisão sistemática aplicada a empresas virtuais (EVs), com o propósito de identificar trabalhos que abordam duas lacunas na área, como por exemplo identificar características compartilhadas e não compartilhadas pelas empresas neste ambiente, e a partir da identificação destas características, os autores identificaram dois tipos de EVs, a hierárquica e a não hierárquica. A EV hierárquica configura-se como um ambiente que apresenta uma empresa líder e é responsável pelo desenvolvimento do produto final. Na EV não hierárquica, os parceiros atuam como uma entidade única e o principal mecanismo de coordenação é a auto-organização. Também foi verificado através da aplicação de um questionário a 18 empresas se as características levantadas com o apoio da literatura são aplicáveis ao cenário real.

Afsarmanesh et al. [6] apresentam uma revisão sobre modelos, metodologias e ferramentas para gerenciar e operar os ambientes de criação de empresas virtuais (ACVs), em que um dos principais problemas é a confiança. Neste caso, a confiança foi definida como uma condição prévia para cooperação, que facilita a seleção de parceiros na criação e configuração de organizações virtuais em ACVs. Entre tantos modelos, metodologias e ferramentas, os autores apresentam classificações e perspectivas para a aplicação da confiança em ambientes de rede de colaboração, bem como um modelo de um sistema para gerenciamento da confiança.

Lee [26] apresenta uma revisão sistemática para as organizações virtuais baseadas no modelo de federação, realizada no contexto do Keystone - serviço de segurança do OpenStack - para verificar as implementações que estão sendo realizadas neste serviço e estão relacionadas com OV, buscando levantar características que são necessárias para aplicar esse conceito em uma OV dinâmica, por exemplo, o uso de uma arquitetura centralizada, questões de autenticação e segurança.

Nikghadam et al. [28] apresentam uma revisão de metodologias que abordam o problema de seleção de parceiros de negócio no ambiente das EVs, contemplando metodologias de tomada de decisão multi-critério, abordagens de otimização e outras abordagens.

Portanto, através dos trabalhos de revisão apresentados, pode-se notar que nenhum aborda uma revisão que busque identificar abordagens envolvendo confiança, reputação e computação em nuvem no ambiente das Organizações Virtuais. Nesse sentido, torna-se interessante verificar como cada conceito é tratado neste ambiente dinâmico das OVs. Por fim, a Seção 6 apresenta a metodologia de revisão proposta para o levantamento de abordagens em 
Uma Revisão Sistemática sobre Abordagens em Organizações Virtuais no Contexto de Confiança, Reputação e Computação em Nuvem

OVs que estejam relacionados com computação em nuvem e outros conceitos como confiança e reputação.

\section{Metodologia de Revisão}

Várias formas de revisão vêm sendo empregadas para sintetizar os estudos primários existentes na literatura, como por exemplo: revisão sistemática, mapeamento sistemático, revisão integrativa, meta-análise, entre outras [29, 30].

Estas formas de revisão utilizam principalmente como fonte de dados a literatura sobre um determinado tema [30], sendo que elas diferem na forma como a pesquisa é conduzida e o objetivo final idealizado. Por exemplo, a revisão sistemática é um estudo em profundidade, ou seja, mais detalhado e específico [29], enquanto o mapeamento sistemático é realizado em largura, ou seja, é mais abrangente e visa entre outras coisas fornecer uma visão geral sobre uma determinada área [31].

De acordo com Kitchenham e Charters [29], a revisão sistemática pode ser definida como um método de pesquisa que tem por objetivo identificar, avaliar e interpretar a literatura relevante a uma ou mais questões de pesquisa ou uma determinada área. De forma mais específica, este tipo de revisão apresenta vários propósitos, sendo os mais comuns definidos como:

- Sumarizar os estudos primários existentes na literatura que estão relacionados à uma determinada área ou questão de pesquisa, com o propósito de identificar formas de aplicação, características, comparações, entre outras;

- Identificação de lacunas no estudos primários para a realização de investigações futuras;

- Construir um embasamento teórico dos estudos primários para, por exemplo, compor o capítulo de trabalhos relacionados de uma dissertação/tese.

Algumas orientações para a realização de uma revisão sistemática, na área da Ciência da Computação, são descritas por Kitchenham e Charters [29], sendo divididas em algumas atividades, como:

- Planejamento: engloba o estudo de viabilidade da revisão, definição de uma ou mais questões de pesquisa, desenvolvimento e validação do protocolo de revisão. O protocolo de revisão, de forma básica, é visto como um passo a passo para a realização da revisão, compreendendo os elementos: mecanismos de busca, critérios e procedimentos de seleção de estudos, estratégia de extração de dados e síntese, entre outros; 
Uma Revisão Sistemática sobre Abordagens em Organizações Virtuais no Contexto de Confiança, Reputação e Computação em Nuvem

- Condução: envolve a busca, seleção e avaliação dos estudos primários para a extração de informações e síntese das informações;

- Relatório: uma vez respondida as questões de pesquisa, deve-se escrever um documento sobre a revisão realizada, podendo ser um artigo, capítulo de dissertação ou tese, entre outros.

Além do modelo de revisão sistemática apresentado por Kitchenham e Charters [29], voltado para a aplicação em engenharia de software, existem outros modelos que podem ser utilizados para o processo de revisão sistemática. É o caso do modelo proposto por Conforto et al. [32] e adotado para a aplicação neste artigo. O modelo proposto por Conforto et al. [32] é composto pelas fases de entrada, processamento e saída, com seus processos detalhados na Figura 2.

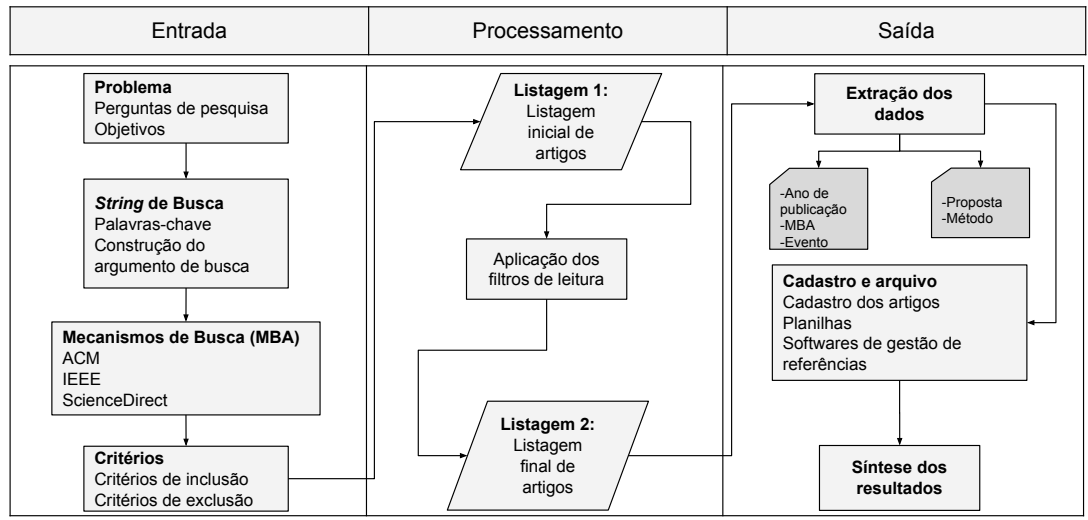

Figura 2. Modelo para condução da revisão sistemática. Adaptado de [32]

A adoção deste modelo de revisão foi baseada em alguns motivos, como por exemplo:

- Destaca-se pelo nível de detalhamento no processo de revisão e em relação a outros métodos voltados exclusivamente ao mapeamento sistemático em engenharia de software, como é o caso do método proposto por Petersen [31];

- É baseado em dois métodos de revisão já existentes, sendo eles: Levy e Ellis [33] e Biolchini et al. [34];

- Pode servir de referências para estudos em outras áreas, através de uma adaptação do roteiro para cada pesquisa realizada. 
Uma Revisão Sistemática sobre Abordagens em Organizações Virtuais no Contexto de Confiança, Reputação e Computação em Nuvem

Por fim, a aplicação do modelo proposto por Conforto et al. [32] é descrita nas próximas seções. A Seção 6.1 apresenta as questões de pesquisa, string de busca, mecanismos de busca acadêmica usados e os critérios de inclusão e exclusão. Por fim, na Seção 6.2, são apresentadas as etapas referentes à condução das buscas bem como a aplicação dos critérios de inclusão e exclusão e os filtros de leitura.

\subsection{Fase de Entrada da Revisão Sistemática}

Por meio do modelo de revisão sistemática adotado, pretende-se responder as seguintes questões de pesquisa:

- Questão Principal (QP):

- QP - Quais são os trabalhos mais recentes que abordam Organizações Virtuais no contexto da Ciência da Computação.

- Questões Secundárias (QS):

- $\mathrm{QS}_{1}$ - Quais são os trabalhos que relacionem organizações virtuais e o problema da confiança?

- $\mathrm{QS}_{2}$ - Quais são os trabalhos que relacionem organizações virtuais e reputação ou sistemas de reputação?

- $\mathrm{QS}_{3}$ - Quais são os trabalhos que apresentem a integração de organizações virtuais e computação em nuvem?

A string de busca representa a expressão, composta por palavras-chave, que será utilizada nos mecanismos de busca acadêmicos (MBA) para a condução das pesquisas. As palavras-chave utilizadas devem estar relacionadas com OV, contemplando virtual organization e sua variação britânica virtual organisation e pelo menos um dos conceitos elencados (trust, confidence, reputation, cloud computing), sendo necessário que estejam presentes nos campos de título, resumo e palavras-chave. Desse modo, a string de busca utilizada é composta por:

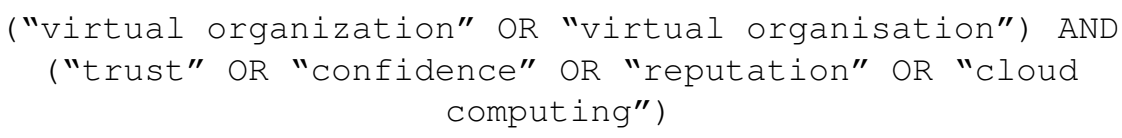

Dada a relevância na área de ciência de computação e no tema pesquisado, os mecanismos de busca acadêmicos (MBAs) utilizados para a condução da revisão sistemática, foram: (a) ACM Digital Library (ACM-DL); (b) IEEE Xplore e (c) Science Direct. 
Uma Revisão Sistemática sobre Abordagens em Organizações Virtuais no Contexto de Confiança, Reputação e Computação em Nuvem

Assim, a Tabela 1 apresenta a aplicação da string de busca nos MBAs sem uso de nenhum filtro e nenhum critério de inclusão/exclusão na busca inicial nos MBAs mencionados acima, retornando um total de 165 artigos.

Tabela 1. Resultados da busca

\begin{tabular}{|c|c|c|c|c|}
\hline & ACM-DL & ScienceDirect & IEEE & Total \\
\hline Inicial & 34 & 34 & 97 & 165 \\
\hline Filtrada & 11 & 11 & 53 & 75 \\
\hline
\end{tabular}

Com o propósito de realizar uma filtragem inicial nos artigos encontrados, é proposta a aplicação dos critérios de inclusão e exclusão. Um trabalho será incluído na análise caso ele venha a atender a todos os critérios de inclusão propostos, e um trabalho será removido da lista, caso atenda pelo menos um dos critérios de exclusão. Dessa forma, na Tabela 2 são expostos os critérios utilizados neste trabalho.

Tabela 2. Critérios de Inclusão e Exclusão

\begin{tabular}{|l|l|}
\hline Critérios de Inclusão & Critérios de Exclusão \\
\hline $\begin{array}{l}\text { CI1 - Artigos disponíveis para download atra- } \\
\text { vés da Universidade do Estado de Santa Catarina } \\
\text { (UDESC) }\end{array}$ & CE1 - Artigos duplicados \\
\hline CI2 - Artigos que estejam no idioma inglês & $\begin{array}{l}\text { CE2 - Artigos não relacionados } \\
\text { com organizações virtuais e confi- } \\
\text { ança, reputação ou computação em } \\
\text { nuvem }\end{array}$ \\
\hline $\begin{array}{l}\text { CI3 - Artigos considerados primários e completos } \\
\text { (artigos que apresentam no mínimo quatro pági- } \\
\text { nas) }\end{array}$ & \\
\hline CI4 - Artigos publicados entre 2010 e 2015 & \\
\hline
\end{tabular}

Para a documentação dos artigos encontrados, são produzidos gráficos com o ano de publicação, MBAs e a proposta do artigo (confiança e OV, reputação e OV e computação em nuvem e OV). Ainda, será apresentada uma síntese de cada trabalho por proposta e método. Para a identificação desses itens, deve-se considerar o título do artigo, resumo e a sua conclusão. Caso surja alguma dúvida durante a leitura desses campos, a leitura completa deve ser realizada para o levantamento de dados. Além das etapas mencionadas, destaca-se que o processo de revisão foi realizado por um único pesquisador, tendo o protocolo e a frase de busca validado pelos demais pesquisadores. 
Uma Revisão Sistemática sobre Abordagens em Organizações Virtuais no Contexto de Confiança, Reputação e Computação em Nuvem

\subsection{Processamento e Análise da Revisão Sistemática}

O processo de busca foi conduzido em um único dia, em junho de 2016, sendo realizado o download da lista de artigos encontrados na busca, usando o formato (BibTeX), possibilitando assim exportar para softwares de gerenciamento de referências bibliográficas como, por exemplo, Mendeley ${ }^{4}$. Deste modo, aplicou-se a string de busca nos MBAs mencionados, o que retornou 75 artigos considerando o filtro de intervalo temporal entre $2010 \mathrm{e}$ 2015, conforme é apresentado na Tabela 1.

Após a condução das buscas nos MBAs, os critérios de inclusão e exclusão foram aplicados. Como é possível verificar na Tabela 3, dos 75 artigos retornados pela busca que possuíam a string de busca em seu título, resumo e palavras-chave, 67 artigos passaram pelos critérios de inclusão e 65 destes não estavam duplicados.

Tabela 3. Aplicação dos critérios de inclusão e exclusão

\begin{tabular}{l|ccc|l}
\hline & ACM-DL & IEEE Xplore & Science Direct & Total \\
\hline Disponíveis & 11 & 53 & 11 & 75 \\
Em Inglês & 11 & 53 & 11 & 75 \\
Completos & 7 & 49 & 11 & 67 \\
$2010-2015$ & 11 & 53 & 11 & 75 \\
\hline Remoção de artigos duplicados - CE1 & 65 \\
Organizações virtuais - CE2 & \multicolumn{2}{|c}{24} \\
\hline
\end{tabular}

A partir dos 65 artigos, aplicou-se o critério de exclusão (CE2) que corresponde aos objetivos da revisão sistemática. Portanto, os filtros de leitura propostos por Conforto et al. [32] foram aplicados para verificar se os artigos são estudos primários e se encaixam nos objetivos e se atendem as perguntas de pesquisa. Ao final, os artigos restantes foram selecionados para a análise dos resultados, sendo que apenas 24 artigos relacionavam-se efetivamente com organizações virtuais e confiança, reputação ou computação em nuvem no contexto da integração.

6.2.1 Análise dos resultados A partir dos 24 artigos resultantes dos filtros de seleção, foram extraídos os dados que atendessem aos objetivos desta revisão. Sendo assim, para cada artigo foram extraídos os dados: ano de publicação, onde foi publicado, evento, se está relacionado com confiança, reputação ou computação em nuvem (classificação) e como o conceito é tratado no artigo.

É possível observar que, conforme o gráfico da Figura 3, os artigos foram publicados

${ }^{4}$ https://www.mendeley.com/ 
Uma Revisão Sistemática sobre Abordagens em Organizações Virtuais no Contexto de Confiança, Reputação e Computação em Nuvem

entre os anos de 2010 e 2014, sendo que entre 2010 e 2013 o número foi o mesmo, 5 artigos, e em 2014 houve uma leve queda.

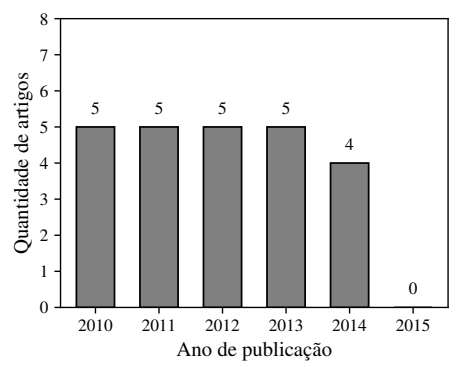

Figura 3. Artigos publicados por ano

Por base de dados, conforme o gráfico da Figura 4, os seis artigos disponíveis no Science Direct (SD) foram publicados em periódicos, os quatro artigos da ACM-DL (ACM) foram publicados em eventos (iiWAS'10, i-KNOW'11, WIMS'13 e UCC 2014) e os 14 artigos do IEEE Xplore (IEEE) foram publicados em eventos (SYNASC 2011, SNPD 2010, INCoS 2010, ISCISC 2012, ISISE 2010, DASC 2011, IC2E 2014, CloudCom 2013, ICACT 2013, ICPP 2012, TrustCom 2012, ICWS 2014, e-Science 2013 e CGC/SCA 2012).

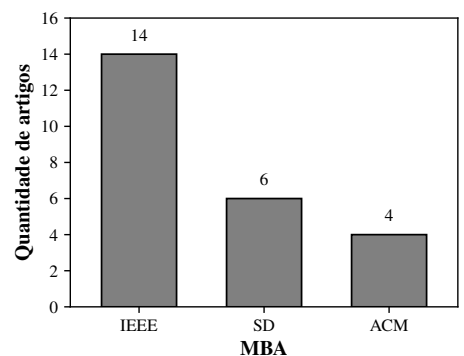

Figura 4. Artigos publicados por base

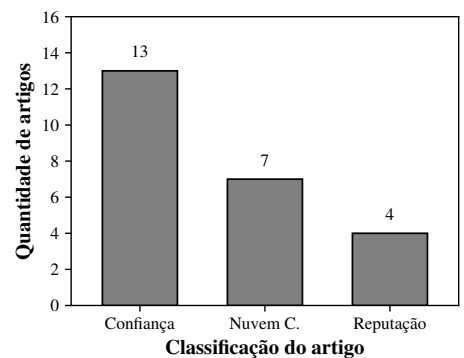

Figura 5. Artigos publicados por tema

Por classificação, de acordo com o gráfico da Figura 5, percebe-se que 13 artigos estão relacionados com OV e confiança, quatro trabalhos são relativos a OV e reputação e sete artigos tratam da integração de OV e computação em nuvem.

Considerando-se que os trabalhos revisados tratam do relacionamento de OV com confiança, reputação e nuvem computacional, nota-se que o maior enfoque entre os trabalhos analisados é o relacionamento de OV e confiança, contando com oito trabalhos, no veículo de publicação mais utilizado (IEEE). Contudo, a quantidade de artigos analisados no período 
Uma Revisão Sistemática sobre Abordagens em Organizações Virtuais no Contexto de Confiança, Reputação e Computação em Nuvem

determinado constitui uma amostra pequena de trabalhos (quando comparada ao trabalho de revisão sistemática realizada por Esposito e Evangelista [1], que analisaram 357 artigos relacionados com Empresas Virtuais (EV)), pode-se concluir que a integração dos conceitos revisados nesse trabalho constitui uma área de pesquisa relativamente nova. Além disto, também pode-se concluir que trata-se de uma área em expansão, dado o número de publicações no decorrer dos anos.

Na Seção 7 é apresentada a síntese dos trabalhos obtidos na etapa de processamento, dividida pelas combinações dos conceitos de OV e confiança, OV e reputação e OV e computação em nuvem.

\section{Análise dos Trabalhos Obtidos}

Nesta seção é apresentada a síntese dos trabalhos selecionados segundo a metodologia apresentada na Seção 6, classificando-os por meio da observação de aspectos como confiança, reputação e computação em nuvem. A confiança pode se apresentar de diversas formas (controle de acesso, estabelecimento da confiança, indicador de confiança, modelo de confiança, entre outros). A reputação pode apresentar-se como um mecanismo para auxiliar o processo de tomada de decisão em uma OV, por exemplo, assessorar o processo de busca e seleção de parceiros. Por sua vez, a computação em nuvem fornece recursos tecnológicos para suportar $\mathrm{o}$ atendimento da oportunidade de colaboração, e os trabalhos analisados abordam aspectos da integração de OVs com computação em nuvem e problemas de segurança e autenticação nestes ambientes.

\subsection{Organizações Virtuais e Confiança}

A confiança é um dos principais problemas envolvidos na OV, pois os membros necessitam compartilhar, integrar e combinar dados e informações. Os trabalhos analisados apresentam modelos de confiança para OV, construção de um indicador de confiança e o uso da confiança como mecanismo de distribuição de recursos computacionais e controle de acesso.

Muitos autores definem modelos de confiança para OVs. Squicciarini et al. [3] apresentam um modelo de confiança que visa estabelecer a confiança em OVs entre os parceiros de negócio da OV e auxiliar nos processos de tomada de decisão. Em Neata et al. [35] é definido um modelo de confiança para agentes, sendo que a confiança é calculada através das características de cada agente e o seu desempenho em participações passadas. Calmet e Maret [36] definem diretrizes para a definição, implementação e geração da confiança em OVs. Kaur e Sengupta [37] apresentam um framework para a modelagem e cálculo da confiança em Grids computacionais, utilizando métricas como a satisfação dos consumidores e 
Uma Revisão Sistemática sobre Abordagens em Organizações Virtuais no Contexto de Confiança, Reputação e Computação em Nuvem

o feedback para gerenciar recursos em Grids. Zhenzhen e Wenzhong [38] apresentam um modelo de confiança dinâmico baseado no comportamento das entidades e analisam os requerimentos do gerenciamento de informações em OVs, cujo estudo do modelo de confiança e suas propriedades podem servir de base para a construção de mecanismos para o controle de acesso.

A confiança também auxilia no controle de acesso a recursos computacionais ou informações em que os mecanismos tradicionais de controle de acesso não são empregados, devido à dinamicidade da $\mathrm{OV}$ e a interação com usuários desconhecidos [39]. Weng et al. [40] empregam o conceito de confiança na troca de arquivos confidenciais e destacam que o relacionamento de confiança entre os parceiros é a base para a colaboração. Karaoglanoglou e Karatza [41] apresentam um mecanismo em que o processo de direcionamento de requisições a provedores confiáveis é feito com base nos valores globais de confiança. Em Nogoorani e Jalili [42] é proposto um framework para controle de acesso a ambientes de Grid Computing, no qual a validação da confiança e o gerenciamento de risco são adicionados aos serviços básicos de controle de acesso disponíveis em uma Grid. Para lidar com usuários não locais e requisições não reconhecidas, a confiança é utilizada para tomar decisões com base no comportamento anterior de cada usuário, devido à dinamicidade da $\mathrm{OV}$.

De outra forma, alguns trabalhos utilizam a confiança como um indicador para a tomada de decisão na OV. Li et al. [43] desenvolveram um modelo de escolha de parceiros baseada em grupo, em que é utilizada a opinião do nó central (ex. gerente da OV), opinião de um terceiro elemento (nós que interagiram com A) e a confiança direta (A confia em B). Li et al. [43] deixam claro que somente a confiança é considerada na etapa de seleção de parceiros, sendo que outras questões são desconsideradas, como a qualidade de serviço. Qi et al. [44] apresentam um indicador de confiança para provedores de nuvem computacional, com base na série histórica de valores de Quality of Service (QoS), que pode ser utilizado para selecionar provedores de nuvem computacional que sejam confiáveis com base na qualidade do serviço que entregam. Ressaltam que fazer a análise pontual (somente em um momento, desconsiderando a série histórica) não é indicada, pois os valores de QoS são flutuantes e dependentes de rede.

Além da aplicabilidade do termo confiança em organizações virtuais conforme os trabalhos apresentados, outros artigos analisados abordam a confiança através de outras visões que não estão relacionadas com modelos e construção da confiança em OVs e o uso do indicador de confiança como mecanismo de distribuição de recursos computacionais. Em Alqithami e Hexmoor [45] são apresentadas técnicas para a criação e utilização do capital social em organizações virtuais e os relacionamentos entre os componentes do capital social, sendo eles valores compartilhados, confiança, valores culturais, laços de rede, normas e procedimentos. Por fim, em Cowles et al. [46] é abordado o gerenciamento da identidade para tratar do problema de risco e confiança em organizações virtuais, em que o modelo de gerencia- 
Uma Revisão Sistemática sobre Abordagens em Organizações Virtuais no Contexto de Confiança, Reputação e Computação em Nuvem

mento da identidade foi baseado nos resultados encontrados em 14 entrevistas realizadas com entidades/pessoas que implementam o gerenciamento da identidade em OVs.

Assim, a formação de uma OV confiável representa diversos desafios, devido à heterogeneidade dos participantes, a falta de uma forma padronizada de estabelecimento de confiança e as próprias limitações da OV, como por exemplo a localização [3].

\subsection{Organizações Virtuais e Reputação}

A reputação no ambiente das OVs pode ser entendida como uma coleção de comentários a respeito das transações realizadas com os membros da rede de colaboração. Dessa forma, os trabalhos analisados apresentam abordagens que utilizam o valor de reputação como um mecanismo para o suporte ao processo de busca e seleção de parceiros, existente durante a criação de uma organização virtual.

Mashayekhy e Grosu [47] apresentam um framework para a criação de OVs utilizando a reputação dos provedores de serviço de grid, para a composição da $\mathrm{OV}$, em que o valor de reputação é calculado com base no histórico de comportamentos desse provedor. Papaioannou e Stamoulis [48] apresentam um sistema de reputação que usa como métrica de reputação o desempenho individual de cada agente em uma OV baseada em grid. Hadjichristofi e Fugini [49] também desenvolvem e aplicam um sistema de reputação para selecionar de forma dinâmica os nós (usuários) que apresentam uma maior reputação, com o propósito de compor a OV e iniciar o processo de colaboração.

Javaid et al. [50] apresentam um sistema de reputação aplicado especificamente para a seleção eficiente de parceiros para a composição de OVs em ambientes de recuperação de desastres e emergências. Neste tipo de ambiente, em virtude da sua dinamicidade, os métodos comuns de seleção não são aplicáveis. O sistema de reputação é apresentado como uma arquitetura orientada a serviço (SOA), composta pelos componentes responsáveis pela coleta, compilação e agregação dos fatores e critérios e outro elemento responsável pelo cálculo geral da reputação em situações de emergência.

\subsection{Organizações Virtuais e Computação em Nuvem}

Um dos diferenciais das redes de colaboração, como por exemplo as organizações virtuais, é o uso de uma tecnologia de comunicação como infraestrutura de comunicação entre os membros. Deste modo, a OV se comunica com outros membros e pode utilizar os recursos computacionais e sistemas fornecidos pela Computação em Nuvem $(\mathrm{CN})$. O uso dos recursos da nuvem visa auxiliar no processo de colaboração, aumentar sua eficiência enquanto almeja diminuir custos e reduzir o tempo de resposta. Ainda, de acordo com Li et al. [23], uma organização virtual baseada na CN deve ser dinâmica, autônoma e distribuída. 
Uma Revisão Sistemática sobre Abordagens em Organizações Virtuais no Contexto de Confiança, Reputação e Computação em Nuvem

Esses fatores trazem desafios para o gerenciamento de segurança nesses ambientes.

Os trabalhos analisados foram divididos em categorias: artigos que tratam da integração de OV com computação em nuvem e artigos que tratam dos problemas de autenticação e segurança nesses ambientes e abordagens que fornecem suporte para plataformas de computação em nuvem (ex.: OpenStack).

A integração entre os conceitos de $\mathrm{OV}$ e $\mathrm{CN}$ traz diversos benefícios para as organizações. Surajbali et al. [19] apresentam um sistema colaborativo, GloNet, que utiliza a CN como base para fornecer acesso descentralizado, de forma remota, a cadeia de suprimentos de uma empresa, em que cada membro da OV pode visualizar, editar e criar produtos, serviços e fluxos de trabalho. Ali et al. [20] apresentam uma abordagem de compartilhamento de recursos computacionais entre os usuários de uma rede social, modelada como uma OV, para que os usuários possam compartilhar seus recursos ociosos com outras pessoas, utilizando mecanismos de confiança e relacionamento existentes nas próprias redes sociais. Outros trabalhos como Huqqani et al. [21] utilizam a combinação dos conceitos para disponibilizar redes neurais como serviço através do ambiente de CN e Langenberg et al. [22] utilizam o modelo de serviço SaaS para a gestão do conhecimento, em ambientes de colaboração, justificando que a nuvem computacional facilita o processo de colaboração, considerando que as entidades estão geograficamente dispersas.

Li et al. [23], apresentam um framework baseado em políticas de segurança e gerenciamento de confiança para prover protocolos flexíveis e dinâmicos para o gerenciamento de OVs em CN, levando em consideração as políticas internas de segurança dos membros da OV.

Outros trabalhos buscam fornecer suporte para a criação de organizações virtuais através de plataformas de CN por exemplo, Openstack, OpenNebula e Eucalyptus. Lee e Desai [24] descrevem um modelo de implementação de OV baseadas na plataforma OpenStack, apresentando um modelo de OV com os seus componentes na nuvem e os problemas que a abordagem busca solucionar, como a autenticação e segurança em ambientes externos, utilizando o serviço de segurança do OpenStack (KeyStone). García et al. [25] apresentam um serviço de filiação de membros da OV para prover federação de identidades em ambientes que apresentam múltiplos provedores. Lee [26] apresenta uma extensão do trabalho realizado em Lee e Desai [24] e uma revisão sistemática para as OVs baseadas no modelo de federação.

Então, as OVs utilizam as tecnologias como forma de suporte a oportunidade de colaboração. Através do uso da computação em nuvem, a OV aumenta sua eficiência e flexibilidade enquanto diminui custos e o tempo de resposta

Ao final, os resultados da revisão sistemática foram sumarizados nas Tabelas 4, 5 e 6. As tabelas apresentam vários critérios que foram analisados e coletados ao longo do processo de revisão sistemática, como por exemplo, os dados referentes ao artigo (autores, 
Uma Revisão Sistemática sobre Abordagens em Organizações Virtuais no Contexto de Confiança, Reputação e Computação em Nuvem

título e ano de publicação), o mecanismo de busca acadêmica, a proposta do artigo (confiança e OV, reputação e OV e computação em nuvem e OV), artigo completo (4 páginas ou mais) e também em qual contexto é aplicado.

O contexto de aplicação foi baseado em cada questão secundária exposta na Seção 6.1. Através disto, notou-se que os conceitos são aplicados por meio de diversas formas na OV, como:

- A confiança apresenta-se como um indicador para a tomada de decisão (apoio à decisão), gerenciamento e alocação de recursos, controle de acesso e gerenciamento de identidades. Além disto, alguns trabalhos apresentam modelos de confiança aplicados nas OVs;

- A reputação auxilia na seleção de parceiros de negócio na formação da OV, alocação de recursos e nas outras fases da OV;

- Já a computação em nuvem e OV, apresenta alguns aspectos relativos a autenticação, segurança e privacidade e outros que são decorrentes desta integração, por exemplo, OV e OpenStack. 
Tabela 4. Resumo dos trabalhos analisados - parte 1/3

\begin{tabular}{|c|c|c|c|c|c|c|}
\hline Autor & Título & Ano & MBA & Páginas & Conceito & Aplicação \\
\hline $\begin{array}{l}\text { Papaioannou e } \\
\text { Stamoulis }\end{array}$ & $\begin{array}{l}\text { Reputation-based estimation of } \\
\text { individual performance in collaborative } \\
\text { and competitive grids }\end{array}$ & 2010 & SD & 7 & Reputação & $\begin{array}{c}\text { Alocação de } \\
\text { Recursos }\end{array}$ \\
\hline $\begin{array}{l}\text { Zhenzhen e } \\
\text { Wenzhong }\end{array}$ & $\begin{array}{c}\text { A Behavior-Based Dynamic Trust Model } \\
\text { of Virtual Organization }\end{array}$ & 2010 & IEEE & 5 & Confiança & $\begin{array}{l}\text { Modelo de } \\
\text { Confiança }\end{array}$ \\
\hline $\begin{array}{l}\text { Hadjichristofi e } \\
\text { Fugini }\end{array}$ & $\begin{array}{l}\text { A dynamic web service-based trust and } \\
\text { reputation scheme for scientific } \\
\text { workflows }\end{array}$ & 2010 & $\mathrm{ACM}$ & 7 & Reputação & $\begin{array}{l}\text { Auxiliar nas } \\
\text { fases da OV }\end{array}$ \\
\hline Weng et al. & $\begin{array}{l}\text { Grid-based collaborative simulation } \\
\text { system for vehicle crashworthiness }\end{array}$ & 2010 & SD & 5 & Confiança & $\begin{array}{l}\text { Apoio à } \\
\text { Decisão }\end{array}$ \\
\hline Li et al. & $\begin{array}{c}\text { CloudVO: Building a Secure Virtual } \\
\text { Organization for Multiple Clouds } \\
\text { Collaboration }\end{array}$ & 2010 & IEEE & 5 & $\begin{array}{l}\text { Computação } \\
\text { em Nuvem }\end{array}$ & $\begin{array}{l}\text { Questões de } \\
\text { segurança e } \\
\text { privacidade }\end{array}$ \\
\hline Squicciarini et al. & $\begin{array}{c}\text { Trust establishment in the formation of } \\
\text { Virtual Organizations }\end{array}$ & 2011 & SD & 10 & Confiança & $\begin{array}{l}\text { Modelo de } \\
\text { Confiança }\end{array}$ \\
\hline Li et al. & $\begin{array}{l}\text { A Group-Choose Model for Partner } \\
\text { Selection in Virtual Organization }\end{array}$ & 2011 & IEEE & 7 & Confiança & $\begin{array}{l}\text { Apoio à } \\
\text { Decisão }\end{array}$ \\
\hline $\begin{array}{l}\text { Karaoglanoglou } \\
\quad \text { et al. }\end{array}$ & $\begin{array}{c}\text { Resource discovery in a Grid system: } \\
\text { Directing requests to trustworthy virtual } \\
\text { organizations based on global trust } \\
\text { values }\end{array}$ & 2011 & SD & 13 & Confiança & $\begin{array}{l}\text { Métrica de } \\
\text { Gerencia- } \\
\text { mento de } \\
\text { Recursos }\end{array}$ \\
\hline Langenberg et al. & $\begin{array}{c}\text { Knowledge management in cloud } \\
\text { environments }\end{array}$ & 2011 & $\mathrm{ACM}$ & 4 & $\begin{array}{l}\text { Computação } \\
\text { em Nuvem }\end{array}$ & $\begin{array}{l}\text { Integração } \\
\text { entre OV e } \\
\text { CN }\end{array}$ \\
\hline
\end{tabular}


Tabela 5. Resumo dos trabalhos analisados - parte $2 / 3$

\begin{tabular}{|c|c|c|c|c|c|c|}
\hline Autor & Título & Ano & MBA & Páginas & Conceito & Aplicação \\
\hline Neata et al. & Trust Model for Virtual Organizations & 2011 & IEEE & 7 & Confiança & $\begin{array}{l}\text { Modelo de } \\
\text { Confiança }\end{array}$ \\
\hline $\begin{array}{l}\text { Mashayekhy e } \\
\text { Grosu }\end{array}$ & $\begin{array}{l}\text { A Reputation-Based Mechanism for } \\
\text { Dynamic Virtual Organization } \\
\text { Formation in Grids }\end{array}$ & 2012 & IEEE & 9 & Reputação & $\begin{array}{l}\text { Formação da } \\
\text { OV }\end{array}$ \\
\hline Kaur e Sengupta & $\begin{array}{l}\text { A Trust Model Based on P2P Trust } \\
\text { Models for Secure Global Grids }\end{array}$ & 2012 & IEEE & 5 & Confiança & $\begin{array}{l}\text { Modelo de } \\
\text { Confiança }\end{array}$ \\
\hline Arasteh et al. & $\begin{array}{l}\text { A trust and reputation-based access } \\
\text { control model for virtual organizations }\end{array}$ & 2012 & IEEE & 6 & Confiança & $\begin{array}{l}\text { Controle de } \\
\text { Acesso }\end{array}$ \\
\hline $\begin{array}{l}\text { Alqithami e } \\
\text { Hexmoor }\end{array}$ & Social Capital in Virtual Organizations & 2012 & IEEE & 5 & Confiança & $\begin{array}{l}\text { Formação da } \\
\text { OV }\end{array}$ \\
\hline Ali et al. & $\begin{array}{l}\text { Social networking for sharing cloud } \\
\text { resources }\end{array}$ & 2012 & IEEE & 6 & $\begin{array}{l}\text { Computação } \\
\text { em Nuvem }\end{array}$ & $\begin{array}{l}\text { Integração } \\
\text { entre OV e } \\
\quad \text { CN }\end{array}$ \\
\hline Cowles et al. & $\begin{array}{c}\text { Identity Management for Virtual } \\
\text { Organizations: An Experience-Based } \\
\text { Model }\end{array}$ & 2013 & IEEE & 6 & Confiança & $\begin{array}{l}\text { Gerenciamento } \\
\text { de Identidade }\end{array}$ \\
\hline Javaid et al. & $\begin{array}{l}\text { A reputation management system for } \\
\text { efficient selection of disaster } \\
\text { management team }\end{array}$ & 2013 & IEEE & 5 & Reputação & $\begin{array}{l}\text { Seleção via } \\
\text { Reputação }\end{array}$ \\
\hline García et al. & $\begin{array}{c}\text { Identity Federation with VOMS in Cloud } \\
\text { Infrastructures }\end{array}$ & 2013 & IEEE & 6 & $\begin{array}{l}\text { Computação } \\
\text { em Nuvem }\end{array}$ & $\begin{array}{l}\text { Autenticação } \\
\text { e Segurança }\end{array}$ \\
\hline
\end{tabular}


Tabela 6. Resumo dos trabalhos analisados - parte $3 / 3$

\begin{tabular}{|c|c|c|c|c|c|c|}
\hline Autor & Título & Ano & MBA & Páginas & Conceito & Aplicação \\
\hline $\begin{array}{l}\text { Nogoorani e } \\
\text { Jalili }\end{array}$ & $\begin{array}{l}\text { TIRIAC: A trust-driven risk-aware } \\
\text { access control framework for Grid } \\
\text { environments }\end{array}$ & 2013 & SD & 16 & Confiança & $\begin{array}{l}\text { Controle de } \\
\text { Acesso }\end{array}$ \\
\hline Calmet e Marret & $\begin{array}{c}\text { Toward a trust model for } \\
\text { knowledge-based communities }\end{array}$ & 2013 & $\mathrm{ACM}$ & 4 & Confiança & $\begin{array}{l}\text { Modelo de } \\
\text { Confiança }\end{array}$ \\
\hline Lee e Desai & $\begin{array}{l}\text { Approaches for Virtual Organization } \\
\text { Support in OpenStack }\end{array}$ & 2014 & IEEE & 6 & $\begin{array}{l}\text { Computação } \\
\text { em Nuvem }\end{array}$ & $\begin{array}{c}\text { OV e } \\
\text { Openstack }\end{array}$ \\
\hline Huqqani et al. & $\begin{array}{l}\text { Novel concepts for realizing neural } \\
\text { networks as services in the sky }\end{array}$ & 2014 & SD & 9 & $\begin{array}{l}\text { Computação } \\
\text { em Nuvem }\end{array}$ & $\begin{array}{l}\text { OV na } \\
\text { Nuvem }\end{array}$ \\
\hline Lee & $\begin{array}{c}\text { A Design Space Review for General } \\
\text { Federation Management Using Keystone }\end{array}$ & 2014 & $\mathrm{ACM}$ & 5 & $\begin{array}{l}\text { Computação } \\
\text { em Nuvem }\end{array}$ & $\begin{array}{c}\text { OV e } \\
\text { Openstack }\end{array}$ \\
\hline Qi et al. & $\begin{array}{c}\text { A Trust Evaluation Method for Cloud } \\
\text { Service with Fluctuant QoS and Flexible } \\
\text { SLA }\end{array}$ & 2014 & IEEE & 7 & Confiança & $\begin{array}{l}\text { Apoio à } \\
\text { Decisão }\end{array}$ \\
\hline
\end{tabular}


Uma Revisão Sistemática sobre Abordagens em Organizações Virtuais no Contexto de Confiança, Reputação e Computação em Nuvem

\section{Conclusão}

Este artigo apresentou uma revisão de trabalhos sobre Organizações Virtuais (OVs) com abordagens sobre confiança, reputação e no contexto da integração com Computação em Nuvem (CN). Dos 75 artigos resultantes do processo de revisão sistemática, apenas 24 foram classificados e categorizados como relevantes para a análise dos resultados. Assim sendo, 13 artigos foram categorizados como relevantes no quesito OVs e confiança, 4 artigos com relevância em OVs e reputação e, por fim, 7 artigos na categoria da integração de OVs com computação em nuvem.

Através da análise do conteúdo dos artigos, pôde-se verificar que os artigos relacionados com o problema da confiança em organizações virtuais estão na maioria das vezes abordando conceitos como o uso da métrica de confiança para o controle de acesso em ambientes distribuídos, enquanto alguns trabalhos tratam da construção ou modelagem de um indicador de confiança para fornecer suporte aos processos existentes no ciclo de vida da OV.

Já nos artigos relacionados com reputação observa-se que a reputação é utilizada principalmente para a etapa de busca e seleção de parceiros de negócio presente na fase de Formação/Criação da OV, sendo que a reputação configura-se como um valor baseado no histórico de desempenho em participações anteriores do provedor de nuvem/parceiro de negócio em uma OV. Porém, nenhum dos trabalhos utilizam os mecanismos de reputação para auxiliar a fase de Operação da OV, na qual é necessário monitorar a reputação/confiança de cada entidade, para que um nível baixo de reputação não seja um impeditivo para o atendimento da oportunidade de colaboração.

Por fim, a integração da $\mathrm{CN}$ com o conceito de OV apresenta-se como uma tecnologia que visa auxiliar o processo de colaboração. Contudo, ela também apresenta problemas em decorrência dessa integração, como por exemplo autenticação, segurança e outros que estão relacionados com a qualidade de serviço fornecida pelas provedores de nuvem. Tal fato motiva a criação de um indicador de confiança, que represente a percepção de confiança na qualidade de serviço que o consumidor possui em relação ao serviço prestado.

Percebeu-se que nenhum dos artigos analisados apresentaram como contribuição a integração dos três conceitos (confiança, reputação e computação em nuvem) com OVs. Ou seja, nenhum trabalho revisado faz uso de provedores de CN para facilitar a colaboração explorando como executar a obtenção, cálculo e gerenciamento de um indicador de confiança na integração de OVs com provedores de $\mathrm{CN}$ e a distribuição desse conhecimento e auxílio nos processos de tomada de decisão existentes no ciclo de vida da OV, através do emprego de um sistema de reputação desse indicador de confiança.

Sendo assim, a revisão sistemática realizada sugere a necessidade de realização de maiores aprofundamentos no campo de pesquisa envolvendo conceitos de confiança, repu- 
Uma Revisão Sistemática sobre Abordagens em Organizações Virtuais no Contexto de Confiança, Reputação e Computação em Nuvem

tação no contexto da integração de $\mathrm{OVs}$ com $\mathrm{CN}$, possibilitando trabalhos que abordem a criação, geração e reputação do indicador de confiança no ambiente da integração, facilitando assim, por exemplo, o processo de busca e seleção de provedores de $\mathrm{CN}$, na fase de criação da OV.

\section{Agradecimentos}

Os autores agradecem à UDESC, ao Conselho Nacional de Desenvolvimento Científico e Tecnológico (CNPq) e ao Programa de Bolsas de Monitoria de Pós-Graduação (PROMOP) pelo suporte financeiro.

\section{Contribuição dos Autores}

- Luís Felipe Bilecki: Pesquisa e escrita do artigo;

- Marcelo da Silva Hounsell: Coordenação do trabalho, correções e direcionamentos de pesquisa;

- Adriano Fiorese: Coordenação do trabalho, correções e direcionamentos de pesquisa.

\section{Referências}

[1] ESPOSITO, E.; EVANGELISTA, P. Investigating virtual enterprise models: Literature review and empirical findings. International Journal of Production Economics, Amsterdam, v. 148, p. 145-157, 2014.

[2] CAMARINHA-MATOS, L. M. et al. Collaborative networked organizations - Concepts and practice in manufacturing enterprises. Computers \& Industrial Engineering, Amsterdam, v. 57, n. 1, p. 46-60, 2009.

[3] SQUICCIARINI, A. C.; PACI, F.; BERTINO, E. Trust establishment in the formation of Virtual Organizations. Computer Standards \& Interfaces, Amsterdam, v. 33, n. 1, p. 13-23, 2011.

[4] PUTNIK, G. D. et al. Virtual enterprise integration: Challenges of a new paradigm. Virtual Enterprise Integration: Technological and Organizational Perspectives: Technological and Organizational Perspectives, Hershey, p. 1, 2005.

[5] JøSANG, A.; ISMAIL, R.; BOYD, C. A survey of trust and reputation systems for 
Uma Revisão Sistemática sobre Abordagens em Organizações Virtuais no Contexto de Confiança, Reputação e Computação em Nuvem

online service provision. Decision Support Systems, Amsterdam, v. 43, n. 2, p. 618 644, 2007. Emerging Issues in Collaborative Commerce.

[6] AFSARMANESH, H.; CAMARINHA-MATOS, L. M.; MSANJILA, S. S. Models, methodologies, and tools supporting establishment and management of secondgeneration VBEs. IEEE Transactions on Systems, Man, and Cybernetics, Part C (Applications and Reviews), New Jersey, v. 41, n. 5, p. 692-710, 2011.

[7] REIN, G. L. Reputation Information Systems : A Reference Model. Sciences-New York, New York, v. 00, n. C, p. 1-10, 2005.

[8] MELL, P. M.; GRANCE, T. SP 800-145. The NIST Definition of Cloud Computing. Technical report, National Institute of Standards \& Technology, Gaithersburg, MD, United States, 2011.

[9] CAMARINHA-MATOS, L. M.; AFSARMANESH, H. Collaborative networks: Reference modeling. New York: Springer US, 2008.

[10] STRAUSAK, N. Resumée of VoTalk. In: SIEBER, P.; GRIESE, J. (Eds.) Organization Virtualness. Proceedings of the VoNet Workshop. Bern, Simowa Verlag Bern: Simowa Verlag, 1998. p. $9-24$.

[11] MSANJILA, S. S.; AFSARMANESH, H. Trust analysis and assessment in virtual organization breeding environments. International Journal of Production Research, Oxfordshire, v. 46, n. 5, p. 1253-1295, 2008.

[12] OLMEDILLA, D. et al. Security and trust issues in semantic grids. In: Semantic Grid: The Convergence of Technologies, 05271., 2005, Dagstuhl. Anais... Dagstuhl: Internationales Begegnungs- und Forschungszentrum für Informatik (IBFI), Schloss Dagstuhl, 2006.

[13] PAN, M.; LI, M.; YU, Y. A group-choose algorithm supporting virtual organization creation for workflow deployment in cloud environment. Concurrency and Computation: Practice and Experience, New Jersey, v. 25, n. 13, p. 1894-1908, 2013.

[14] CHO, J.-H.; SWAMI, A.; CHEN, I.-R. A survey on trust management for mobile ad hoc networks. IEEE Communications Surveys \& Tutorials Tutorials, NJ, v. 13, n. 4, p. 562-583, 2011.

[15] KO, R. K. L. et al. TrustCloud: A framework for accountability and trust in cloud computing. Proceedings - 2011 IEEE World Congress on Services, SERVICES 2011, New Jersey, p. 584-588, 2011.

[16] SABATER, J.; SIERRA, C. Review on computational trust and reputation models. Artificial Intelligence Review, New York, v. 24, n. 1, p. 33-60, 2005. 
Uma Revisão Sistemática sobre Abordagens em Organizações Virtuais no Contexto de Confiança, Reputação e Computação em Nuvem

[17] RESNICK, P. et al. Reputation systems. Communications of the ACM, New York City, v. 43, n. 12, p. 45-48, 2000.

[18] ZHANG, Q.; CHENG, L.; BOUTABA, R. Cloud computing: State-of-the-art and research challenges. Journal of Internet Services and Applications, New York, v. 1, n. 1, p. $7-18,2010$.

[19] SURAJBALI, B. et al. A cloud-based approach for collaboration of serviced-enhanced products. In: IEEE International Conference on Industrial Engineering and Engineering Management, 8., 2014, Bandar Sunway. Anais... Bandar Sunway: IEEE, 2014. p. 12951299.

[20] ALI, Z.; RASOOL, R. U.; BLOODSWORTH, P. Social networking for sharing cloud resources. In: Second International Conference on Cloud and Green Computing (CGC), 2., 2012, Xiangtan. Anais... Xiangtan: IEEE, 2012. p. 160-166.

[21] HUQQANI, A. A.; MANN, E.; SCHIKUTA, E. Novel concepts for realizing neural networks as services in the sky. Procedia Computer Science, Cairns, v. 29, p. 23152324, 2014.

[22] LANGENBERG, D.; KIND, C.; DAMES, M. Knowledge management in cloud environments. In: 11th International Conference on Knowledge Management and Knowledge Technologies, 11., 2011, Graz. Anais... Graz: ACM, 2011.

[23] LI, J.; LI, B.; DU, Z.; MENG, L. CloudVO: Building a Secure Virtual Organization for Multiple Clouds Collaboration. 11th ACIS International Conference on Software Engineering Artificial Intelligence Networking and Parallel/Distributed Computing (SNPD), London, p. 181-186, 2010.

[24] LEE, C. A.; DESAI, N. Approaches for Virtual Organization Support in OpenStack. In: IEEE International Conference on Cloud Engineering (IC2E), 7., 2014, Boston. Anais... Boston: IEEE, 2014. p. 432-438.

[25] GARCÍA, Á. L.; FERNÁNDEZ-DEL CASTILLO, E.; PUEL, M. Identity Federation with VOMS in Cloud Infrastructures. In: IEEE 5th International Conference on Cloud Computing Technology and Science (CloudCom), 5., 2013, Bristol. Anais... Bristol: IEEE, 2013. v. 1. p. 42-48.

[26] LEE, C. A. A design space review for general federation management using keystone. In: IEEE/ACM 7th International Conference on Utility and Cloud Computing (UCC), 7., 2014, London. Anais... London: IEEE, 2014. p. 720-725.

[27] NAMI, M. R. Virtual Organizations: An Overview. In: Intelligent Information Processing IV. Boston: Springer, 2008. p. 211-219. 
Uma Revisão Sistemática sobre Abordagens em Organizações Virtuais no Contexto de Confiança, Reputação e Computação em Nuvem

[28] NIKGHADAM, S. et al. A survey of partner selection methodologies for virtual enterprises and development of a goal programming-based approach. The International Journal of Advanced Manufacturing Technology, p. 1-22, 2015.

[29] KITCHENHAM, B.; CHARTERS, S. Guidelines for performing systematic literature reviews in software engineering. Technical Report Technical report, Ver. 2.3 EBSE Technical Report. EBSE, Keele, UK, Keele University and Durham, UK, University of Durham, 2007.

[30] SAMPAIO, R. F.; MANCINI, M. C. Estudos de revisão sistemática: um guia para síntese criteriosa da evidência científica. Braz. J. Phys. Ther.(Impr.), v. 11, n. 1, p. 83-89, 2007.

[31] PETERSEN, K. et al. Systematic Mapping Studies in Software Engineering. In: 12th International Conference on Evaluation and Assessment in Software Engineering, 12., Bari. Anais... Swindon: BCS Learning \& Development Ltd., 2008. p. 68-77.

[32] CONFORTO, E. C.; AMARAL, D. C.; SILVA, S. L. D. Roteiro para revisão bibliográfica sistemática: aplicação no desenvolvimento de produtos e gerenciamento de projetos. In: Congresso Brasileiro de Gestão de Desenvolvimento de Produtos, 8., Porto Alegre. Anais... Porto Alegre: IGDP, 2011.

[33] LEVY, Y.; ELLIS, T. J. A Systems Approach to Conduct an Effective Literature Review in Support of Information Systems Research. Informing Science Journal, v. 9, p. 181212, 2006.

[34] DE ALMEIDA BIOLCHINI, J. C. et al. Scientific research ontology to support systematic review in software engineering. Advanced Engineering Informatics, v. 21, n. 2, p. $133-151,2007$.

[35] NEATA, S.; URZICA, A.; FLOREA, A. M. Trust model for virtual organizations. In: 13th International Symposium on Symbolic and Numeric Algorithms for Scientific Computing (SYNASC), 13., 2011, Timisoara. Anais... Timisoara: IEEE, 2011. p. 357-364.

[36] CALMET, J.; MARET, P. Toward a trust model for knowledge-based communities. In: 3rd International Conference on Web Intelligence, Mining and Semantics, 2013, Madrid. Anais... New York: ACM, 2013. p. 47.

[37] KAUR, D.; SENGUPTA, J. A trust model based on p2p trust models for secure global grids. In: 11th International Conference on Trust, Security and Privacy in Computing and Communications, 11., 2012, Liverpool. Anais... Liverpool: IEEE, 2012. p. 11031108 . 
Uma Revisão Sistemática sobre Abordagens em Organizações Virtuais no Contexto de Confiança, Reputação e Computação em Nuvem

[38] ZHENZHEN, C.; WENZHONG, T. A behavior-based dynamic trust model of virtual organization. In: International Symposium on Information Science and Engineering (ISISE), 2., 2010, Shangai. Anais... Shangai: IEEE, 2010. p. 391-395.

[39] ARASTEH, M.; AMINI, M.; JALILI, R. A trust and reputation-based access control model for virtual organizations. In: 9th International ISC Conference on Information Security and Cryptology (ISCISC), 9., 2012, Tabriz. Anais... IEEE, 2012. p. 121-127.

[40] WENG, Y.; JIN, X.; ZHAO, Z.; CAO, Y.; WANG, J. Grid-based collaborative simulation system for vehicle crashworthiness. Simulation Modelling Practice and Theory, v. 18, n. 6, p. 752-767, 2010.

[41] KARAOGLANOGLOU, K.; KARATZA, H. Resource discovery in a grid system: Directing requests to trustworthy virtual organizations based on global trust values. Journal of Systems and Software, v. 84, n. 3, p. 465-478, 2011.

[42] NOGOORANI, S. D.; JALILI, R. Tiriac: A trust-driven risk-aware access control framework for grid environments. Future Generation Computer Systems, v. 55, p. 238$254,2013$.

[43] LI, M.; YU, Y.; HUANG, Z. A group-choose model for partner selection in virtual organization. In: 9th International Conference on Dependable, Autonomic and Secure Computing (DASC), 9., 2011, Sydney. Anais... Sydney: IEEE, 2011. p. 674-681.

[44] QI, L.; DOU, W.; NI, J.; XIA, X.; MA, C.; LIU, J. A Trust Evaluation Method for Cloud Service with Fluctuant QoS and Flexible SLA. In: International Conference on Web Services (ICWS), 21., 2014, Anchorage. Anais... Anchorage: IEEE, 2014. p. $345-352$.

[45] ALQITHAMI, S.; HEXMOOR, H. Social capital in virtual organizations. In: 4th International Conference on Intelligent Networking and Collaborative Systems (INCoS), 4., 2012, Bucharest. Anais... Bucharest: IEEE, 2012. p. 682-687.

[46] COWLES, R.; JACKSON, C.; WELCH, V. Identity management for virtual organizations: An experience-based model. In: 9th International Conference on eScience (eScience), 9., 2013, Beijing. Anais... Beijing: IEEE, 2013. p. 278-284.

[47] MASHAYEKHY, L.; GROSU, D. A reputation-based mechanism for dynamic virtual organization formation in grids. Proceedings of the International Conference on Parallel Processing, p. 108-117, 2012.

[48] PAPAIOANNOU, T. G.; STAMOULIS, G. D. Reputation-based estimation of individual performance in collaborative and competitive grids. Future Generation Computer Systems, v. 26, n. 8, p. 1327-1335, 2010. 
Uma Revisão Sistemática sobre Abordagens em Organizações Virtuais no Contexto de Confiança, Reputação e Computação em Nuvem

[49] HADJICHRISTOFI, G. C.; FUGINI, M. A dynamic web service-based trust and reputation scheme for scientific workflows. Proceedings of the 12th International Conference on Information Integration and Web-based Applications \& Services - iiWAS '10, p. 56, 2010.

[50] JAVAID, S.; MAJEED, A.; AFZAL, H. A reputation management system for efficient selection of disaster management team. In: 15th International Conference on Advanced Communication Technology (ICACT), 15., 2013, PyeongChang, South Korea. Anais... Piscataway, New Jersey, US: IEEE. p. 829-834. 